\title{
An Unusual Complication of Histoacryl Injection
}

$\mathrm{N}$-Butyl-2-cyanoacrylate (Histoacryl) has been used successfully to control bleeding from esophagogastric varices since the first report in 1986 by Soehendra et al. (1). Complications of Histoacryl injection include local sloughing with ulcer formation (2), rebleeding, sepsis, mediastinitis, esophageal strictures (3) and fever (4). Systemic embolization, including pulmonary and cerebral embolization, have also been reported (5).

We describe here the presentation of an unusual complication of Histoacryl injection. A 42-year-old man presented with a massive attack of hematemesis. Endoscopy revealed bleeding esophageal varices and a nonbleeding gastric varix. Sclerotherapy was carried out until complete obliteration of the esophageal varices was achieved. Later, the patient developed recurrent attacks of melena, and the source was traced to the gastric varix. In our department, injection of the gastric varix was carried out using Histoacryl mixed with Lipiodol (ratio 1:1), with complete solidification of the varix. During the procedure, the patient was sedated with midazolam $5 \mathrm{mg}$, and was irritable and experienced some choking while being moved into the supine position. Four days later, when he returned for follow-up, the patient was found to be feverish $\left(40^{\circ} \mathrm{C}\right)$, with coughing and blood-streaked sputum. Empirical antibiotics and antipyretics were administered, to no avail. A chest radiograph was taken, showing opacification of the right middle lobe bronchus with Lipiodol (Figure 1). A CT examination showed that the bronchus was patent, with no evidence of lung collapse. Culture and sensitivity tests were done, and the proper antibiotic was prescribed, after which the fever improved over one week. Serial radiographic studies were carried out, and the opacification gradually subsided to normal within nine months.

In our department, 119 patients were injected with Histoacryl during the previous year, and the most common complication observed was fever lasting for a few hours in $90 \%$ of cases.

Aspiration represents one of the unusual complications of Histoacryl injection, and must be borne in mind when dealing with prolonged post-sclerotherapy fever, or if the patient develops respiratory symptoms after injection sclerotherapy.

M. Naga, A. Foda

Dept. of Medicine, Kasr El-Eini Hospital, University of Cairo, Cairo, Egypt
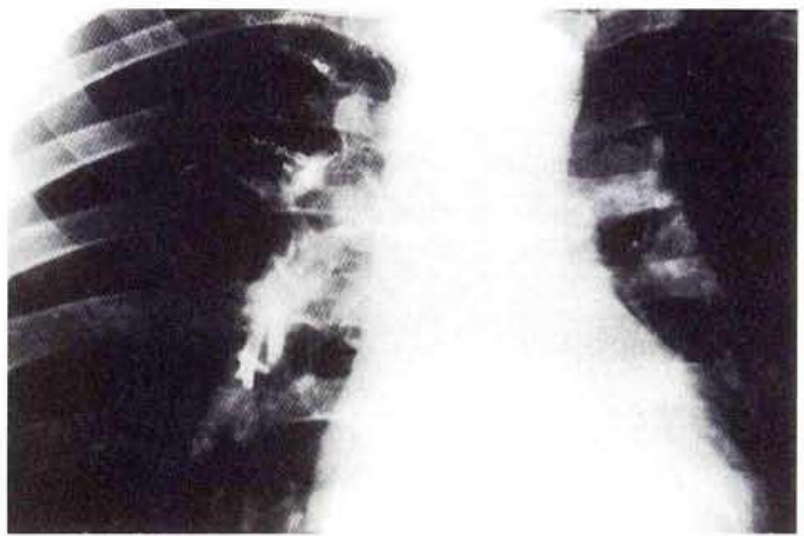

Figure 1: Plain chest radiograph, showing opacification of the right middle lobe bronchus with Lipiodol.

\section{References}

1. Soehendra N, Nan VC, Grim H, et al. Endoscopic obliteration of large esophagogastric varices with Bucrylate. Endoscopy 1986; 18: 25-6.

2. Fabiani B, Degott C, Ramond MJ, et al. Endoscopic obturation of oesophagogastric varices with Bucrylate, 2: morphological study based on 12 autopsy cases. Gastroenterol Clin Biol 1986; 10: $580-3$.

3. Ramond MJ, Valla D, Gotlib JP, et al. Endoscopic obturation of oesophagogastric varices with Bucrylate, 1: clinical study of 49 patients. Gastroenterol Clin Biol 1986; 10: 575-9.

4. Naga M, El-Fiki A. Classification and management of gastric varices. Sci J Cai Med Synd 1993; 5: 137-47.

5. See A, Florent C, Lamy F, et al. Cerebrovascular accidents after endoscopic obliteration of oesophageal varices with isobutyl-2-cyanoacrylate in 2 patients. Gastroenterol Clin Biol 1986; 10: $604-7$.

Corresponding Author

M. Naga, M.D., 15 Rushdy St.

Abdeen, Cairo, Egypt

Fax: $+20-2-961078$ 